\title{
Synthesis of Poly(vinyl chloride) and Silica Gel Polymer Hybrids via $\mathrm{CH} / \pi$ Interaction
}

\author{
Takeru IwAmURA, Kaoru ADACHI, and Yoshiki CHUJO ${ }^{\dagger}$ \\ Department of Polymer Chemistry, Graduate School of Engineering, Kyoto University, \\ Katsura, Nishikyo-ku, Kyoto 615-8510, Japan
}

(Received February 25, 2004; Accepted September 2, 2004; Published November 15, 2004)

\begin{abstract}
Organic-inorganic polymer hybrids were prepared utilizing the $\mathrm{CH} / \pi$ interactions. The sol-gel reaction of alkoxysilanes such as tetramethoxysilane (TMOS), methyltrimethoxysilane (MeTMOS), and phenyltrimethoxysilane (PhTMOS) was carried out in the presence of the polymer having the activated $\mathrm{CH}$ groups such as poly(vinyl chloride) (PVC). It was found that the transparent and homogeneous polymer hybrid was obtained from PVC and the alkoxysilane having aromatic groups such as PhTMOS. The transparent and homogeneous polymer hybrids could be obtained in a wide range of mass ratios $\left(\mathrm{PVC} / \mathrm{PhSiO}_{1.5}=9 / 1-3 / 7\right)$. In these polymer hybrids, the $\mathrm{CH} / \pi$ interaction was found to act as an important role for the dispersion of PVC in the silica matrix. [DOI 10.1295/polymj.36.871] KEY WORDS

Interaction /
\end{abstract}

Preparation of organic-inorganic polymer hybrids from organic polymer and alkoxysilanes has been intensely investigated by many researchers. ${ }^{1-13}$ In hybridization, the sol-gel reaction of alkoxysilanes is the most typical technique for the synthesis of these hybrid materials. This sol-gel reaction consists of three unit reactions (Scheme 1). When alkoxysilanes are used as precursors, $\mathrm{Si}-\mathrm{OH}$ groups are formed by hydrolysis of alkoxy silane groups (step 1). Subsequently, condensation of the hydroxyl groups leads to a formation of a Si-O-Si linkage (step 2). With further hydrolysis and condensation, a siloxane network is developed via cross-linking of the oligomers (step 3). The advantage of the sol-gel technique for the preparation of composite materials is the fact that the reactions can be carried out at ambient temperature, while the conventional melt fusion technique for silica glasses requires much higher temperature.
Thus, it enables an introduction of organic elements into inorganic materials without deteriorating their functionalities.

We have recently investigated organic-inorganic polymer hybrids, in which organic polymers are dispersed in a silica gel matrix at the molecular level. ${ }^{14,15}$ However, we have mainly used strong intermolecular interactions such as hydrogen bonding interaction and have applied hydrophilic polymers such as poly(vinyl alcohol), poly( $N$-vinylpyrrolidone), and poly(2-methyl-2-oxazoline) for hybridization. Therefore, we have little knowledge about hybridization using hydrophobic polymers. In the previous study, we have prepared the organic-inorganic polymer hybrids from polystyrene and alkoxysilanes having aromatic ring such as phenyltrimethoxysilane (PhTMOS). ${ }^{16}$ Transparent and homogeneous polymer hybrids could be obtained, in which the $\pi-\pi$ interactions between phenyl groups

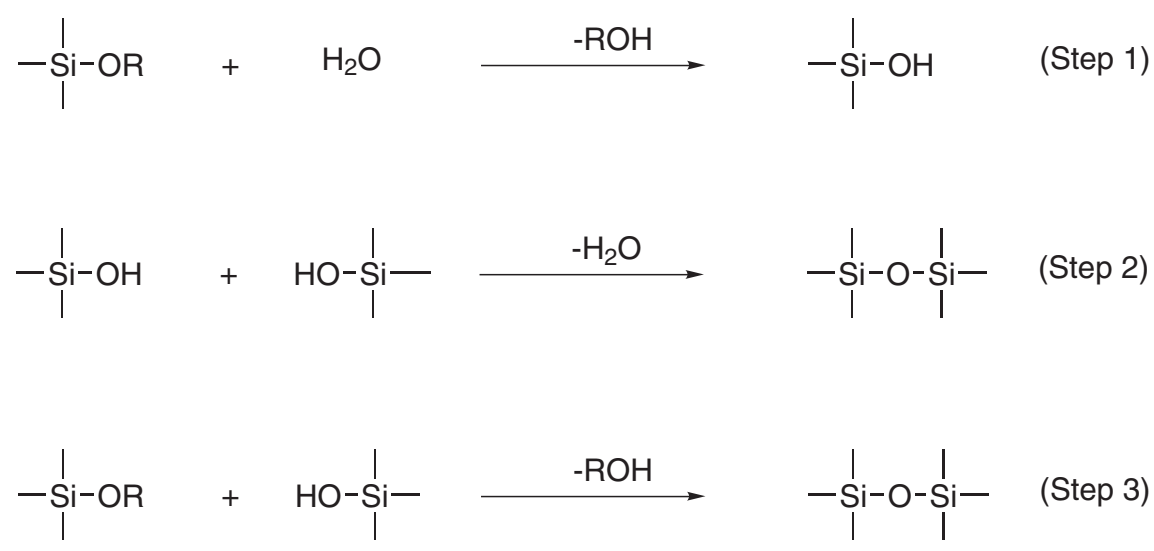

Scheme 1. Sol-gel reaction of alkoxysilane.

${ }^{\dagger}$ To whom correspondence should be addressed (E-mail: chujo@chujo.synchem.kyoto-u.ac.jp). 

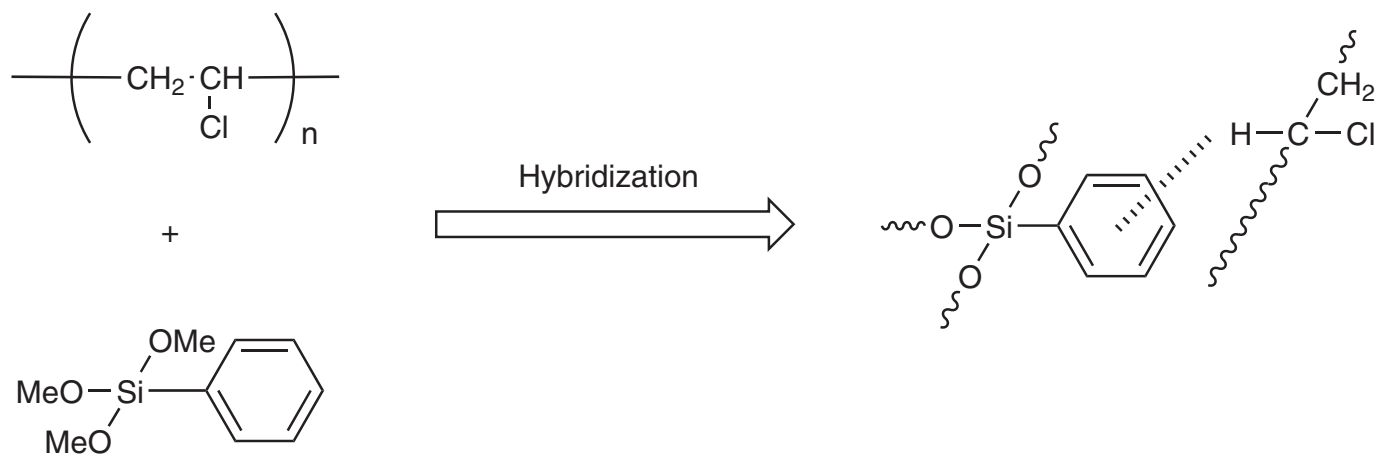

Scheme 2. Hybridization of PVC and PhTMOS.

of the organic polymer and silica gel were found to play a critical role for the homogeneity. It is important to explore new interactions between organic component and inorganic one for the preparation of various kinds of homogeneous polymer hybrids.

In this article, we report detail results on the synthesis of organic-inorganic polymer hybrids based on the $\mathrm{CH} / \pi$ interaction, which is weaker than the $\pi-\pi$ interaction (Scheme 2). The utilization of $\mathrm{CH} / \pi$ interactions might provide an extension of new hydrophobic polymer hybrids.

\section{EXPERIMENTAL}

\section{Materials}

Poly(vinyl chloride) (PVC) $(\mathrm{DP}=1100)$ was purchased from Wako Pure Chemical Industries, Ltd. PVC was purified by reprecipitation from its THF solution into methanol and dried in vacuo. Tetramethoxysilane (TMOS) and phenyltrimethoxysilane (PhTMOS) were purchased from Tokyo Kasei Kogyo Co., Ltd. Methyltrimethoxysilane (MeTMOS), cyclohexyltrimethoxysilane (cyclohexylTMOS), tolyltrimethoxysilane (TolTMOS) were purchased from Chisso Corporation. Mesityltrimetoxysilane (MesTMOS) was synthesized by the following method. All alkoxysilanes were distilled and stored under nitrogen. Tetrahydrofuran (THF) was dried over sodium and distilled under nitrogen. The other solvents and reagents were used as supplied.

\section{Measurements}

The FT-IR spectra were obtained on a PerkinElmer 1600 infrared spectrometer. Scanning electron microscopy (SEM) measurements were conducted using a JEOL JSM-5600B system. Thermogravimetric analysis (TGA) was performed using a TG/DTA6200, SEIKO Instruments, Inc., with heating rate of $10^{\circ} \mathrm{C} /$ min in air. Differential scanning calorimetry (DSC) thermograms were recorded with a DSC200, SEIKO Instruments, Inc., with a heating rate of $10^{\circ} \mathrm{C} / \mathrm{min}$ under nitrogen atmosphere. Dynamic mechanical analy- sis (DMA) was conducted on a DMS 210 (SEIKO Instruments, Inc.). The sample was measured at $1 \mathrm{~Hz}$. The temperature for the measurement was raised from 20 to $180^{\circ} \mathrm{C}$ at the rate of $2^{\circ} \mathrm{C} / \mathrm{min}$.

\section{Synthesis of Mesityltrimethoxysilane (MesTMOS)}

Distilled mesityl bromide $(6.51 \mathrm{~g}, 32.8 \mathrm{mmol})$ was dissolved in dry $\mathrm{Et}_{2} \mathrm{O}(30 \mathrm{~mL})$ with magnesium $(0.874 \mathrm{~g}, 36.1 \mathrm{mmol})$ at room temperature in nitrogen. The mixture was stirred at room temperature for $2 \mathrm{~h}$ and refluxed for $1 \mathrm{~h}$ and was added dropwise to $\mathrm{Et}_{2} \mathrm{O}$ $(40 \mathrm{~mL})$ solution of TMOS $(4.97 \mathrm{~g}, 32.8 \mathrm{mmol})$ at $0{ }^{\circ} \mathrm{C}$. The mixture was stirred for $44 \mathrm{~h}$ in nitrogen. During the stirring, the reaction temperature was gradually raised to room temperature. White solids were removed by filtration and the solution was evaporated to dryness. MesTMOS was purified by distilled the obtained oily product. Yield: $3.86 \mathrm{~g}$ (49\%). Bp: $87^{\circ} \mathrm{C} / 1.1 \mathrm{mmHg} .{ }^{1} \mathrm{H}$ NMR $\left(\mathrm{CDCl}_{3}, 400 \mathrm{MHz}\right): \delta$ $2.29\left(\mathrm{~s}, 3 \mathrm{H}, \mathrm{CH}_{3}-\mathrm{C}_{6} \mathrm{H}_{2}\left(\mathrm{CH}_{3}\right)_{2}-\right), 2.52\left(\mathrm{~s}, 6 \mathrm{H}, \mathrm{CH}_{3}-\right.$ $\left.\mathrm{C}_{6} \mathrm{H}_{2}\left(\mathrm{CH}_{3}\right)_{2}-\right), 3.63$ (s, 9H, MesSi $\left.\left(\mathrm{OCH}_{3}\right)_{3}\right), 6.87$ (s, 2H, $\left(\mathrm{CH}_{3}\right)_{3}-\mathrm{C}_{6} \underline{\mathrm{H}}_{2}-$ ). ${ }^{13} \mathrm{C}$ NMR (DMSO- $d_{6}, 22.4$ $\mathrm{MHz}): \delta 21.0,23.4,50.0,124.0,128.8,140.0,145.9$. $\mathrm{EI} / \mathrm{MS} m / z 240[\mathrm{M}]^{+}$.

Synthesis of Organic-Inorganic Polymer Hybrids from PVC and Alkoxysilanes (Typical Procedure)

PVC $(0.5 \mathrm{~g})$ was dissolved in $20 \mathrm{~mL}$ of THF with the prescribed amounts of TMOS (1.27 g), MeTMOS $(1.14 \mathrm{~g})$, cyclohexylTMOS (0.78g), PhTMOS (0.78 g), TolTMOS $(0.76 \mathrm{~g})$, MesTMOS $(0.70 \mathrm{~g})$ and 0.1 $\mathrm{M}$ aqueous $\mathrm{HCl}$ solution (10 equiv to alkoxysilane). After being stirred at room temperature for $1 \mathrm{~h}$, the mixture was placed in a polypropylene vessel covered with a wiping paper and left in air at $40^{\circ} \mathrm{C}$ for 2 weeks. The obtained polymer hybrid was dried in vacuo at $60^{\circ} \mathrm{C}$ for $2 \mathrm{~d}$.

\section{RESULTS AND DISCUSSION}

The effect of PhTMOS content on the homogeneously of polymer hybrid was first investigated. PVC 
Table I. Synthesis of PVC and alkoxysilane polymer hybrids

\begin{tabular}{|c|c|c|c|c|c|c|c|c|c|}
\hline \multirow{2}{*}{ Run } & \multirow{2}{*}{$\begin{array}{c}\text { PVC } \\
(\mathrm{g})\end{array}$} & \multicolumn{2}{|c|}{$\mathrm{R}-\mathrm{Si}(\mathrm{OMe})_{3}$} & \multirow{2}{*}{$\begin{array}{c}\text { Feed ratio of } \\
\mathrm{PVC} \mathrm{PhSiO}_{1.5} \\
(\mathrm{w} / \mathrm{w})\end{array}$} & \multirow{2}{*}{ Appearance } & \multicolumn{3}{|c|}{ Ceramic Yield $(\%)^{\mathrm{a}}$} & \multirow{2}{*}{$T_{\mathrm{d} 10}$} \\
\hline & & $\mathrm{R}-$ & (g) & & & obs. & cal. & obs./cal. & \\
\hline 1 & 0.500 & $\mathrm{MeO}-$ & 1.270 & $1 / 1$ & turbid & 37.8 & 50.1 & 0.75 & 241.9 \\
\hline 2 & 0.504 & Me- & 1.141 & $1 / 1$ & turbid & 40.2 & 47.2 & 0.85 & 241.4 \\
\hline 3 & 0.500 & $\mathrm{Ph}-$ & 0.776 & $1 / 1$ & transparent & 21.9 & 23.4 & 0.94 & 265.5 \\
\hline 4 & 0.510 & Cyclohexyl- & 0.784 & $1 / 1$ & turbid & 18.8 & 22.4 & 0.84 & 257.2 \\
\hline 5 & 0.501 & Tol- & 0.757 & $1 / 1$ & transparent & 22.2 & 21.2 & 1.05 & 264.6 \\
\hline 6 & 0.508 & Mes- & 0.699 & $1 / 1$ & transparent & 19.9 & 17.4 & 1.14 & 248.9 \\
\hline
\end{tabular}

Conditions: PVC and PhTMOS were dissolved in $20 \mathrm{~mL}$ of THF with $0.1 \mathrm{M}$ aq. $\mathrm{HCl}$ (10 eq.). After the mixture was placed at $40{ }^{\circ} \mathrm{C}$ for 2 weeks, the resulting hybrid was evaporated to dryness at $60^{\circ} \mathrm{C}$ for $48 \mathrm{~h}$ under redused pressure.

${ }^{a}$ Ceramic yield $=$ (weight percent of ceramics in the polymer hybrids observed by TGA)/(calculated value of weight percent of ceramics).

(a)

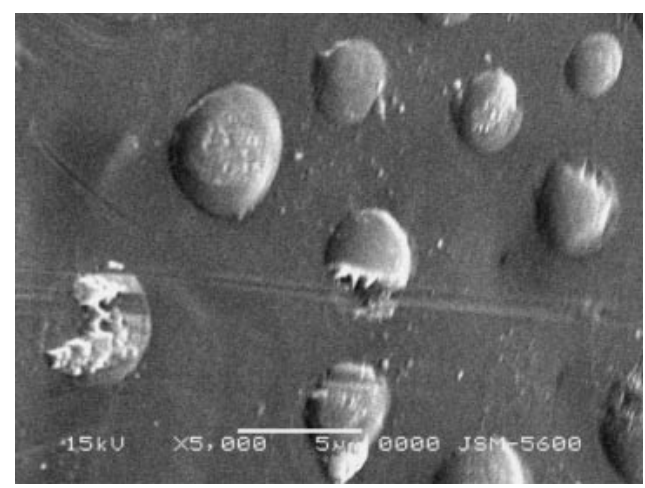

(c)

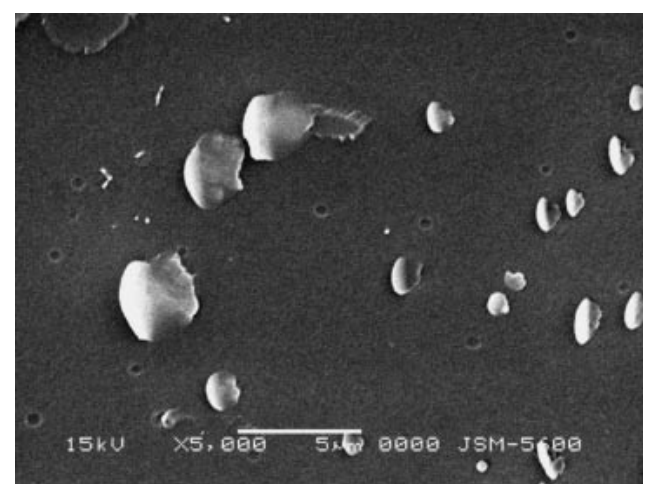

(b)

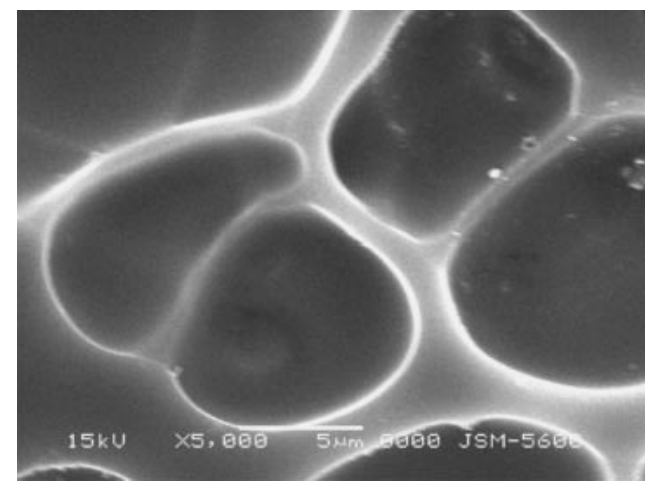

(d)

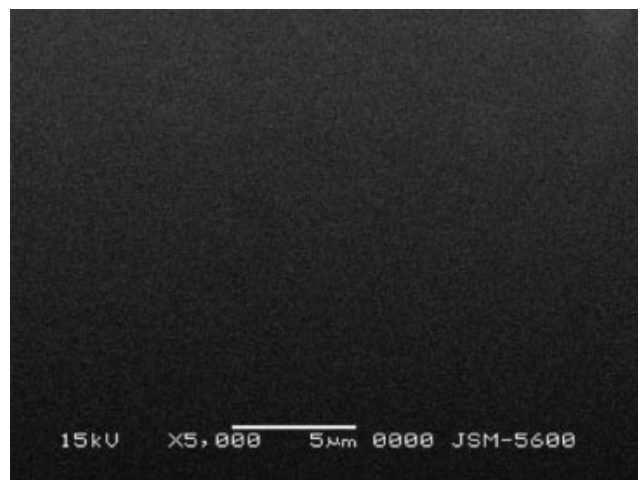

Figure 1. SEM images of the composites from (a) PVC and TMOS (Table I, Run 1), (b) PVC and MeTMOS (Table I, Run 2) (c) PVC and cyclohexylTMOS (Table I, Run 3) and SEM images of the polymer hybrids from (d) PVC and PhTMOS (Table I, Run 4).

and silica gel polymer hybrids were prepared by utilizing a sol-gel reaction of six kinds of alkoxysilanes, TMOS, MeTMOS, cyclohexylTMOS, PhTMOS, TolTMOS, and MesTMOS. The sol-gel reaction proceeds via hydrolysis and condensation of alkoxysilane. The results are summarized in Table I. Transparent and homogeneous polymer hybrids were obtained when arylalkoxysilane was used as the starting alkoxysilane. The dispersity in the resulting poly- mer hybrid was also examined by SEM. As shown in Figure 1a-c, the samples prepared from non-aromatic alkoxysilanes such as TMOS, MeTMOS, and cyclohexylTMOS showed phase separation of silica and the organic polymer. The spherical particles indicating silica were observed in a domain size much lager than $1 \mu \mathrm{m}$. On the other hand, when the samples were prepared from arylalkoxysilane such as PhTMOS, TolTMOS, and MesTMOS, silica domains 
(a)

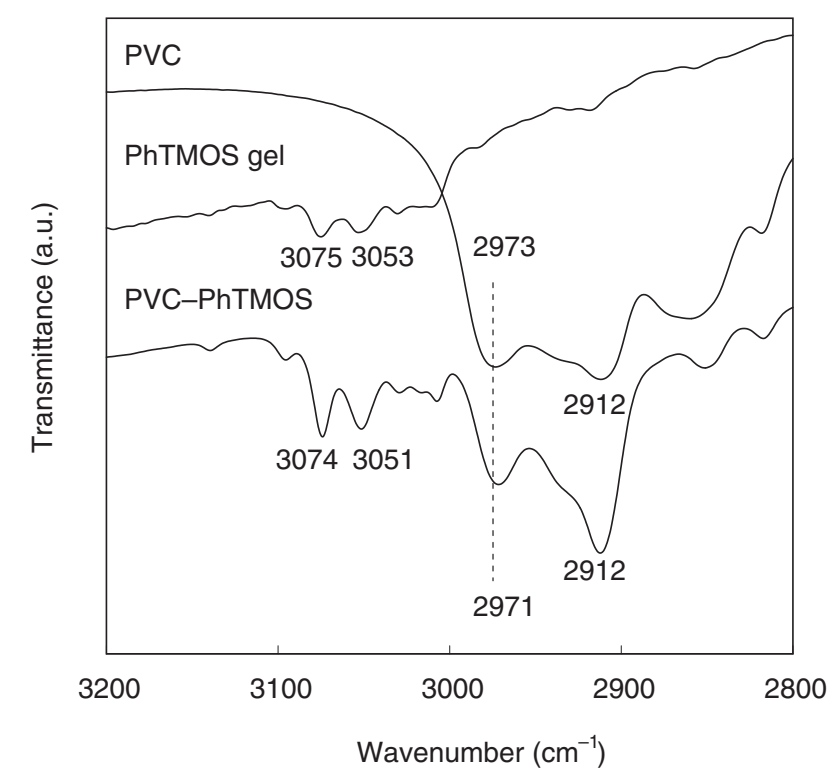

(b)

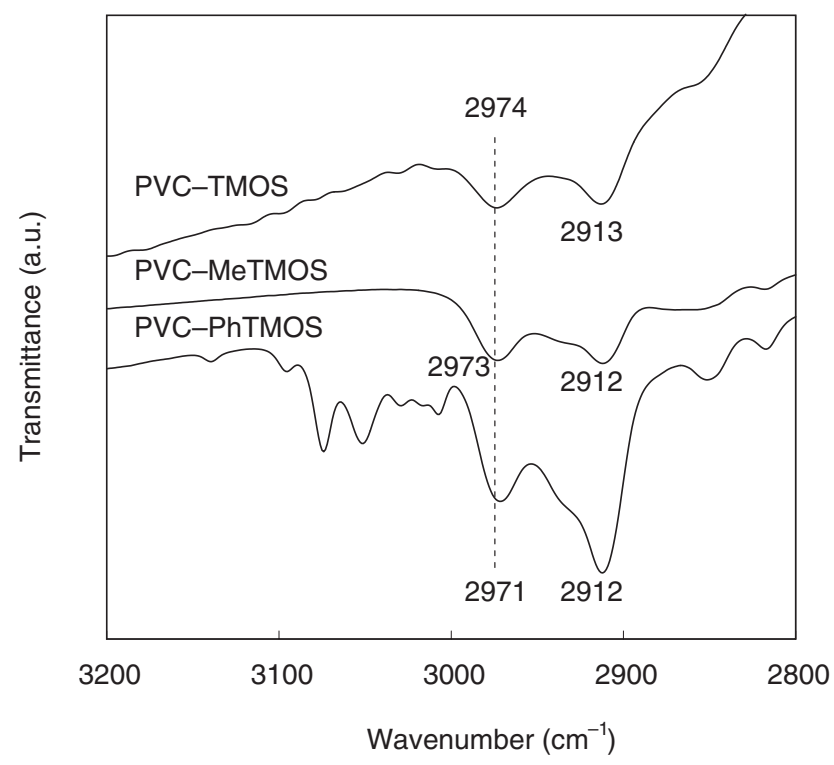

Figure 2. IR spectra of (a) PVC, PhTMOS gel, and PVCPhTMOS hybrid (Table I, Run 3) and (b) PVC-TMOS composite (Table I, Run 1), PVC-MeTMOS composite (Table I, Run 2), and PVC-PhTMOS hybrid (Table I, Run 3).

could not found in a micron order. Figure 1d shows transparent polymer hybrid prepared from PhTMOS. These results indicate that $\mathrm{CH} / \pi$ interactions are quite effective for the synthesis of homogeneous polymer hybrids of polymers having active $\mathrm{CH}$ groups and silica gel.

The FT-IR spectra of PVC, PhTMOS gel, ${ }^{17}$ and PVC-PhTMOS hybrid are shown in Figure 2a. The IR spectrum of PVC showed the absorption band at $2973 \mathrm{~cm}^{-1}$ based on the $\mathrm{CH}$ stretching frequency. In the IR spectrum of PVC-PhTMOS hybrid, this absorption band was observed at $2971 \mathrm{~cm}^{-1}$. In contrast (a)

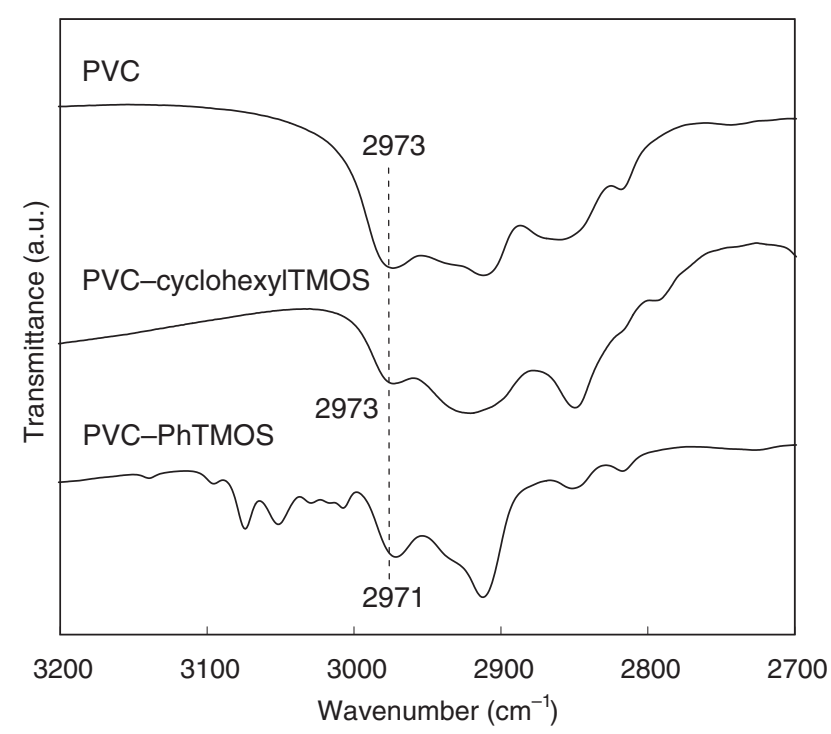

(b)

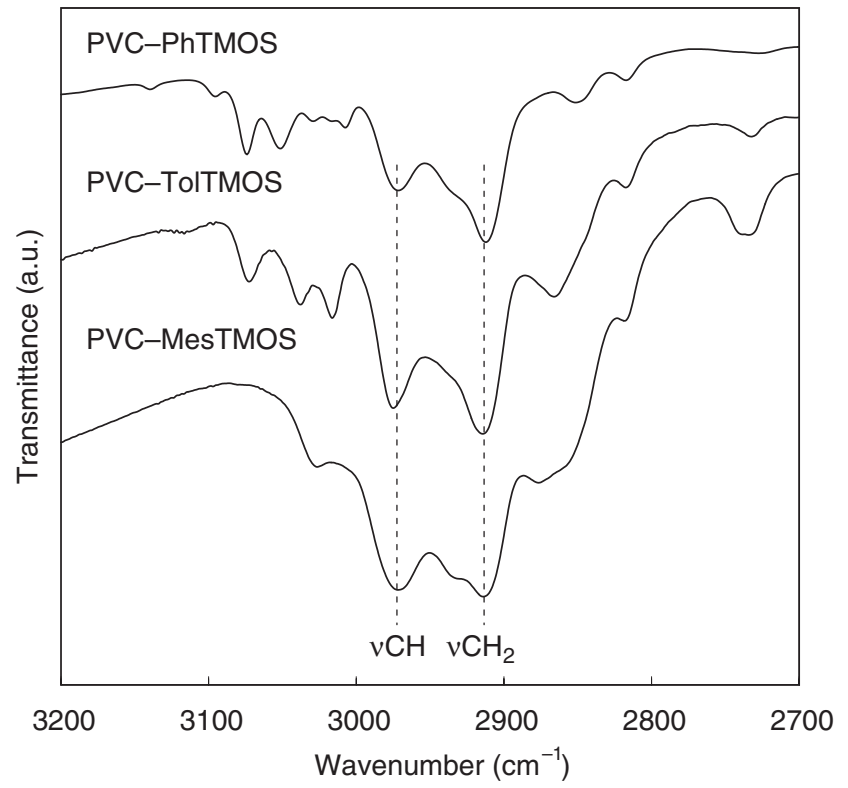

Figure 3. IR spectra of (a) PVC, PVC-cyclohexylTMOS composite (Table I, Run 3), and PVC-PhTMOS hybrid (Table I, Run 4) and (b) PVC-PhTMOS hybrid (Table I, Run 4), PVCTolPhTMOS hybrid (Table I, Run 5), and PVC-MesTMOS hybrid (Table I, Run 6).

with the $\mathrm{CH}$ stretching frequency, the $\mathrm{CH}_{2}$ stretching frequency at $2912 \mathrm{~cm}^{-1}$ was almost unaffected by hybridization. Additionally, the aromatic $\mathrm{CH}$ stretching vibrations at 3075 and $3053 \mathrm{~cm}^{-1}$ were shifted to lower frequency after hybridization (3074 and 3051 $\mathrm{cm}^{-1}$ ). These results may indicate an existence of $\mathrm{CH} / \pi$ interaction between methine proton adjacent to the chlorine atom and phenyl group adjacent to the silicon atom. In the case of the transparent hybrid prepared from PVC and PhTMOS, the $\mathrm{CH}$ stretching frequency was shifted to lower frequency after hybrid- 
Table II. Effect of PhTMOS contents on the homogeneity

\begin{tabular}{|c|c|c|c|c|c|c|c|c|c|}
\hline \multirow{2}{*}{ Run } & \multirow{2}{*}{$\begin{array}{l}\text { PVC } \\
(\mathrm{g})\end{array}$} & \multirow{2}{*}{$\begin{array}{l}\text { PhTMOS } \\
\quad(\mathrm{g})\end{array}$} & \multirow{2}{*}{$\begin{array}{l}\mathrm{HCl} \text { aq. } \\
\quad(\mathrm{mL})\end{array}$} & \multirow{2}{*}{$\begin{array}{c}\text { Feed ratio of } \\
\text { PVC/PhSiO1.5 } \\
(\mathrm{w} / \mathrm{w})\end{array}$} & \multirow{2}{*}{ Appearance } & \multicolumn{3}{|c|}{ Ceramic Yield $(\%)^{\mathrm{a}}$} & \multirow{2}{*}{$\begin{array}{l}T_{\mathrm{d} 10} \\
\left({ }^{\circ} \mathrm{C}\right)\end{array}$} \\
\hline & & & & & & obs. & cal. & obs./cal. & \\
\hline 1 & 1.007 & 0.000 & 0.00 & $10 / 0$ & transparent & 0.0 & 0.0 & 0.00 & 246.6 \\
\hline 2 & 0.907 & 0.156 & 0.14 & $9 / 1$ & transparent & 5.1 & 4.7 & 1.08 & 252.6 \\
\hline 3 & 0.803 & 0.324 & 0.28 & $8 / 2$ & transparent & 8.8 & 9.7 & 0.90 & 258.0 \\
\hline 4 & 0.702 & 0.460 & 0.42 & $7 / 3$ & transparent & 12.3 & 13.9 & 0.89 & 264.7 \\
\hline 5 & 0.608 & 0.641 & 0.56 & $6 / 4$ & transparent & 17.6 & 19.0 & 0.93 & 265.2 \\
\hline 6 & 0.502 & 0.775 & 0.70 & $5 / 5$ & transparent & 23.6 & 23.3 & 1.01 & 267.6 \\
\hline 7 & 0.404 & 0.939 & 0.84 & $4 / 6$ & transparent & 26.2 & 28.0 & 0.93 & 263.7 \\
\hline 8 & 0.301 & 1.073 & 0.98 & $3 / 7$ & transparent & 33.0 & 32.5 & 1.01 & 278.8 \\
\hline 9 & 0.201 & 1.235 & 1.11 & $2 / 8$ & turbid & 33.0 & 37.2 & 0.89 & 282.2 \\
\hline 10 & 0.101 & 1.406 & 1.25 & $1 / 9$ & turbid & 40.0 & 41.9 & 0.95 & 419.7 \\
\hline
\end{tabular}

Conditions: PVC and PhTMOS were dissolved in $20 \mathrm{~mL}$ of THF with $0.1 \mathrm{M}$ aq. $\mathrm{HCl}$ (10 eq.). After the mixture was placed at $40{ }^{\circ} \mathrm{C}$ for 2 weeks, the resulting hybrid was evaporated to dryness at $60^{\circ} \mathrm{C}$ for $48 \mathrm{~h}$ under redused pressure.

${ }^{a}$ Ceramic yield $=$ (weight percent of ceramics in the polymer hybrids observed by TGA)/(calculated value of weight percent of ceramics).

ization. However, in the case of the turbid composites prepared from PVC and TMOS or MeTMOS, this frequency did not change after hybridization (Figure $2 b$ ).

The FT-IR spectra of PVC, PVC-cyclohexylTMOS composite, and PVC-PhTMOS hybrid are shown in Figure 3a. The IR spectra of PVC and PVC-cyclohexylTMOS composite showed the $\mathrm{CH}$ stretching absorption band at $2973 \mathrm{~cm}^{-1}$. In the IR spectrum of PVCPhTMOS hybrid, this absorption band was shifted toward $2971 \mathrm{~cm}^{-1}$. That is, when the polymer hybrids were prepared from alkoxysilanes having phenyl group, the $\mathrm{CH}$ stretching frequency was shifted to lower frequency. Moreover, since ceramic yield do not differ greatly by Table I, Runs 3 and 4, the reactivity of PhTMOS and cyclohexylTMOS were almost same. Therefore, the transparency and homogeneity of polymer hybrids may not depend on the chemical reactivity of PhTMOS and cyclohexylTMOS.

Karatsu et al. reported that the peak intensity of the $\mathrm{CH}$ stretching vibration by forming $\mathrm{CH} / \pi$ interaction increase with increasing the electron density of the aromatic ring in the IR spectra. ${ }^{18}$ Since the more electron-rich $\pi$ system acts as a stronger hydrogen acceptor in forming $\mathrm{CH} / \pi$ interaction, the ratio of the absorption intensities of the two $\mathrm{CH}$ bands assigned to the methine $\left(v_{\mathrm{CH}}\right)$ and methylene $\left(v_{\mathrm{CH}_{2}}\right)$ was expected to increase with increasing the electron density of the aromatic ring. Actually, in Figure $3 b$, the $v_{\mathrm{CH}} /$ $\nu_{\mathrm{CH}_{2}}$ ratios were increase in the order of PVCPhTMOS (0.67), PVC-TolTMOS (0.81), and PVCMesTMOS (0.84). This result suggested that the $\mathrm{CH} / \pi$ interaction act as an important role for the formation of transparent and homogeneous polymer hybrids.

The transparency and homogeneity could be affected by varying the ratio of inorganic element in the polymer hybrids. The effect of PhTMOS content on the homogeneity of the polymer hybrids is summarized in Table II. The feed ratio of $\mathrm{PVC} / \mathrm{PhSiO}_{1.5}$ was varied from $1 / 9$ to $10 / 0$. In the cases of runs $1-8$, the optical appearance of all the samples was transparent. These results suggest that the organic and inorganic phases were mixed at the level smaller than the wave length of visible light, that is, around 200 $\mathrm{nm}$. The appearance of the samples was all turbid with the $\mathrm{PhSiO}_{1.5}$ content higher than $80 \%$. These results suggest that the obtained polymer hybrid became optically turbid owing to the phase separation of the organic and inorganic phases at a scale larger than the wavelength of visible light. The mechanical and thermal properties might be also affected by varying the ratio of inorganic element in the polymer hybrids.

The polymer content calculated was almost the same as that measured from TGA analysis in air. This fact means that the sol-gel reaction was accelerated completely (Table II). TGA profiles of the obtained polymer hybrids are shown in Figure 4. In the case of PVC, $T_{10}$ was observed at $246.6^{\circ} \mathrm{C}$, and in the polymer hybrids (Table II, Runs 2-9) $T_{10}$ was shifted to higher temperature. These results show that the thermal stability was increased with an increase of inorganic content.

The dynamic visco-elasticity of the polymer hybrids was investigated using dynamic mechanical analysis (DMA) to evaluate the effect of PhTMOS content. The results of DMA for the polymer hybrids are shown in Figure 5. In the case of PVC, the value of $E^{\prime}$ was decreased in one step (Figure 5a), while in the case of polymer hybrids, the value of $E^{\prime}$ was decreased in two stages (PVC 60\% and 80\%). This change can be identified more clearly from the peak positions in $\tan \delta$ profiles in Figure 5b. In the case of 


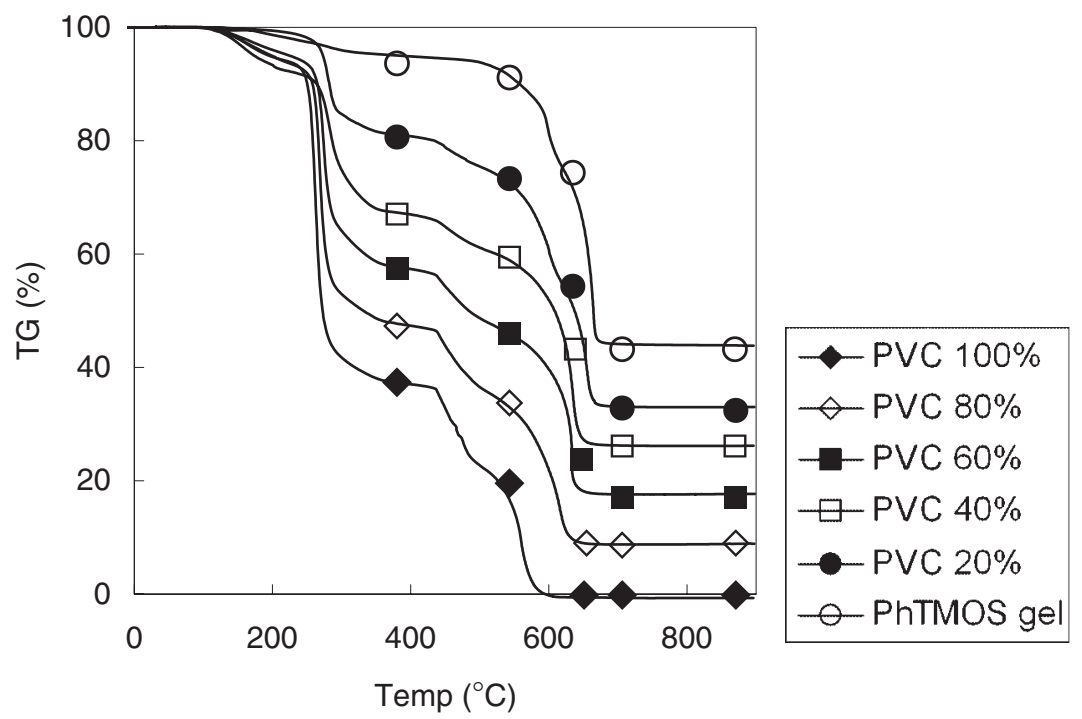

Figure 4. TGA profiles of PVC 100\% (Table II, Run 1), PVC 80\% (Table II, Run 3), PVC 60\% (Table II, Run 5), PVC 40\% (Table II, Run 7), PVC 20\% (Table II, Run 9), PhTMOS gel.

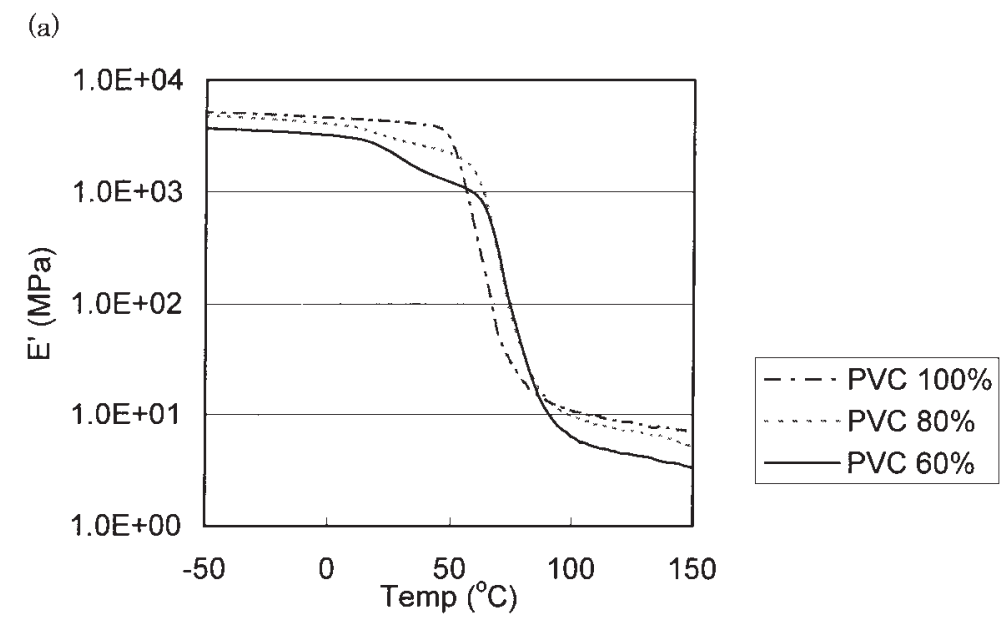

(b)

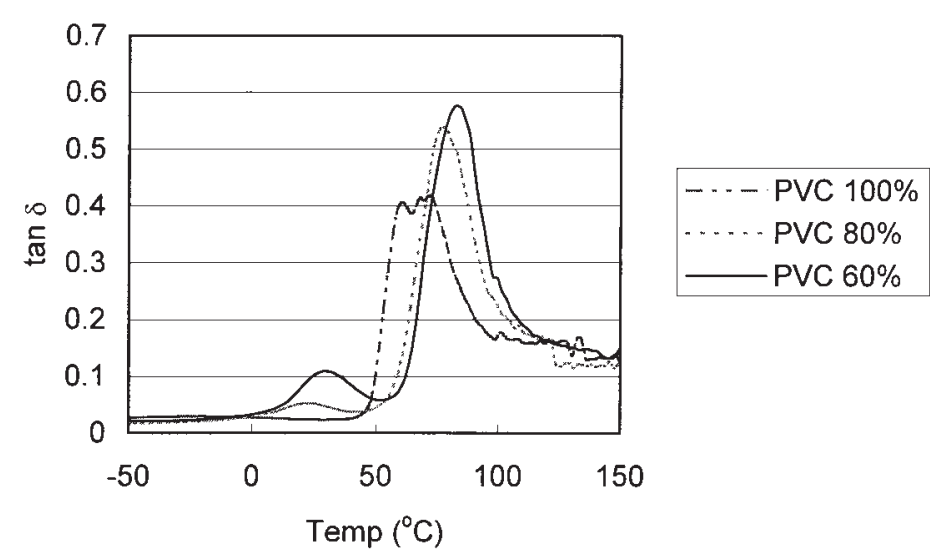

Figure 5. DMA results of PVC 100\% (Table II, Run 1), PVC 80\% (Table II, Run 3), PVC 60\% (Table II, Run 5): (a) profiles of $E^{\prime} v s$. temperature; (b) profiles of $\tan \delta$ s. temperature.

PVC, only one peak was observed. However, in the case of polymer hybrids, there are two peaks observed. The glass transition temperature $\left(T_{\mathrm{g}}\right)$ of the
PhTMOS gel was found at approximately $20-30{ }^{\circ} \mathrm{C}$ in DSC thermograms. That is, two peaks attributable to glass transitions of PhTMOS gel and PVC, respec- 
tively, were observed at a low-temperature region (approximately $0-50{ }^{\circ} \mathrm{C}$ ) and a high temperature region $\left(-50^{\circ} \mathrm{C}\right)$ in Figure $5 \mathrm{~b}$. These results suggest that the phase separation might take place below nanometer order, it should be concluded that although the polymer and silica were distributed uniformly on the naked eye and the SEM level. Moreover, according to the more hybridization, these two peaks were shifted to the higher temperatures. That is, the polymer hybrids had the character of higher glass transition temperatures by means of hybridization.

\section{CONCLUSIONS}

Organic-inorganic polymer hybrids were prepared utilizing the $\mathrm{CH} / \pi$ interactions between the $\mathrm{CH}$ groups of the organic polymer and the phenyl groups of the sol-gel matrix. Transparent and homogeneous polymer hybrids could be obtained by means of acidcatalyzed sol-gel reaction of PhTMOS in the presence of the polymer having the activated $\mathrm{CH}$ groups such as PVC. From the viewpoint of the hybrid preparation, many kinds of hydrophobic polymers are applicable to hybridization by using $\mathrm{CH} / \pi$ interaction.

\section{REFERENCES}

1. G. S. Sur and J. E. Mark, Eur. Polym. J., 21, 1051 (1985).

2. J. E. Mark and G. S. Sur, Polym. Bull., 14, 324 (1985).

3. S. J. Clarson and J. E. Mark, Polym. Commun., 28, 249 (1987).
4. C.-C. Sun and J. E. Mark, Polymer, 30, 104 (1989).

5. J. E. Mark, CHEMTECH, April, 230 (1989).

6. C. J. Wung, Y. Pang, P. N. Prasad, and F. E. Karasz, Polymer, 32, 605, (1991).

7. S. Motakef, T. Suratwala, R. L. Roncone, J. M. Boulton, G. Teowee, G. F. Neilson, and D. R. Uhlmann, J. Non-Cryst. Solids, 178, 31 (1994).

8. S. Motakef, T. Suratwala, R. L. Roncone, J. M. Boulton, G. Teowee, G. F Neilson, and D. R. Uhlmann, J. Non-Cryst. Solids, 178, 37 (1994).

9. M. Yoshida and P. N. Prasad, Appl. Opt., 35, 1500 (1996).

10. C. Xu, L. Eldada, C. Wu, R. A. Norwood, L. W. Shacklette, J. T. Yardley, and Y. Wei, Chem. Mater., 8, 2701 (1996).

11. B. C. Dave, B. Dunn, J. S. Valentine, and J. I. Zink, Anal. Chem., 66, 1120 (1994).

12. C. Claude, B. Garetz, Y. Okamoto, and S. Tripathy, Mater. Lett., 14, 336 (1992).

13. B. L-Davies, M. Samoc, and M. Woodruff, Chem. Mater., 8, 2586 (1996).

14. Y. Chujo and T. Saegusa, Adv. Polym. Sci., 100, 11 (1992).

15. Y. Chujo, in "Organic/Inorganic Polymer Hybrids," J. C. Salamone, Ed., Polymeric Materials Encyclopedia, CRC Press, Boca Raton, FL, 1996, p 4793.

16. R. Tamaki, K. Samura, and Y. Chujo, Chem. Commun., 10, 1131 (1998).

17. PhTMOS $(0.78 \mathrm{~g}$ ) and $0.1 \mathrm{M}$ aqueous $\mathrm{HCl}$ solution (10 equiv to alkoxysilane) were dissolved in $20 \mathrm{~mL}$ of THF. After being stirred at room temperature for $1 \mathrm{~h}$, the mixture was placed in a polypropylene vessel covered with a wiping paper and left in air at $40^{\circ} \mathrm{C}$ for 2 weeks. The obtained PhTMOS gel was dried in vacuo at $60^{\circ} \mathrm{C}$ for $2 \mathrm{~d}$.

18. M. Karatsu, H. Suezawa, K. Abe, M. Hirota, and M. Nishio, Bull. Chem. Soc. Jpn., 59, 3529 (1986). 